\title{
Intraoperative subcortical mapping of a language-associated deep frontal tract connecting the superior frontal gyrus to Broca's area in the dominant hemisphere of patients with glioma
}

\author{
Masazumi Fujii, MD, PhD, ${ }^{1}$ Satoshi Maesawa, MD, PhD, ${ }^{2}$ Kazuya Motomura, MD, PhD, ${ }^{1}$ \\ Miyako Futamura, ST, BAPSY, ${ }^{3}$ Yuichiro Hayashi, PhD, ${ }^{4}$ Itsuko Koba, ST, ${ }^{1}$ \\ and Toshihiko Wakabayashi, MD, PhD'
}

\begin{abstract}
1Department of Neurosurgery, Graduate School of Medicine; ${ }^{2}$ Brain and Mind Research Center; ${ }^{3}$ Department of Rehabilitation; and ${ }^{4}$ Information and Communications Headquarters, Nagoya University, Nagoya, Japan
\end{abstract}

\begin{abstract}
OBJECT The deep frontal pathway connecting the superior frontal gyrus to Broca's area, recently named the frontal aslant tract (FAT), is assumed to be associated with language functions, especially speech initiation and spontaneity. Injury to the deep frontal lobe is known to cause aphasia that mimics the aphasia caused by damage to the supplementary motor area. Although fiber dissection and tractography have revealed the existence of the tract, little is known about its function. The aim of this study was to determine the function of the FAT via electrical stimulation in patients with glioma who underwent awake surgery.
\end{abstract}

METHODS The authors analyzed the data from subcortical mapping with electrical stimulation in 5 consecutive cases ( 3 males and 2 females, age range 40-54 years) with gliomas in the left frontal lobe. Diffusion tensor imaging (DTI) and tractography of the FAT were performed in all cases. A navigation system and intraoperative MRI were used in all cases. During the awake phase of the surgery, cortical mapping was performed to find the precentral gyrus and Broca's area, followed by tumor resection. After the cortical layer was removed, subcortical mapping was performed to assess language-associated fibers in the white matter.

RESULTS In all 5 cases, positive responses were obtained at the stimulation sites in the subcortical area adjacent to the FAT, which was visualized by the navigation system. Speech arrest was observed in 4 cases, and remarkably slow speech and conversation was observed in 1 case. The location of these sites was also determined on intraoperative MR images and estimated on preoperative MR images with DTI tractography, confirming the spatial relationships among the stimulation sites and white matter tracts. Tumor removal was successfully performed without damage to this tract, and language function did not deteriorate in any of the cases postoperatively.

CONCLUSIONS The authors identified the left FAT and confirmed that it was associated with language functions. This tract should be recognized by clinicians to preserve language function during brain tumor surgery, especially for tumors located in the deep frontal lobe on the language-dominant side.

http://thejns.org/doi/abs/10.3171/2014.10.JNS14945

KEY WORDS awake surgery; glioma; subcortical mapping; frontal aslant tract; oncology

$\mathrm{W}$ HITE matter in the deep frontal lobe is known to be associated with language functions, especially the initiation and spontaneity of speech. Injury to the white matter in this area is known to cause aphasia that mimics the aphasia caused by damage to the supplementary motor area (SMA).${ }^{17}$ Recently, a tract that connects the inferior frontal gyrus (IFG) and the superior fron- tal gyrus (SFG) was named "the frontal aslant tract" (FAT) by Catani et al. ${ }^{3}$ Existence of this tract had been reported previously based on diffusion tensor imaging (DTI) and white matter dissection studies. . $^{7,12,15,18}$ This tract has an oblique course from the medial superior to the inferior lateral region, which is how it earned the name frontal aslant tract. ${ }^{3}$ The tract is known to be lateralized to the left hemi-

ABBREVIATIONS DTI = diffusion tensor imaging; ECoG = electrocorticography; FAT = frontal aslant tract; IFG = inferior frontal gyrus; IFOF = inferior frontooccipital fascicle; SFG = superior frontal gyrus; SLF = superior longitudinal fascicle; SMA = supplementary motor area.

SUBMITTED April 28, 2014. ACCEPTED October 13,2014. 
sphere; thus, it is speculated that it may have languagerelated functions. ${ }^{3,12}$ Catani et $a l .{ }^{4}$ have since reported a correlation between damage to this tract and verbal fluency deficits in patients with primary progressive aphasia.

Although the dominant FAT is assumed to be related to language, ${ }^{3,4,12}$ exploration of its function has just begun. In general, the function of white matter pathways is not as well understood as the cortex. One reason for this is related to the limitations of lesion studies, in that it is usually difficult to find a homogeneous patient population with lesions localized to the white matter of interest. Additionally, although the cortices can be mapped by surface electrodes, for example, as a method of preoperative study in patients undergoing epilepsy and brain tumor surgeries, subcortical fibers are not easy to evaluate in this setting. Awake surgery for brain tumors such as gliomas is an important and effective method for resecting as much of the tumor as possible, while also preserving neurological functions. ${ }^{5,15}$ This type of surgery allows us to identify the functions of white matter fibers, as well as define functional boundaries for the resection..$^{5,15}$ Here, we report that subcortical mapping by direct electrical stimulation successfully identified the FAT in patients with brain tumors during image-guided awake surgery.

\section{Methods}

We investigated 5 consecutive patients ( 3 males and 2 females) who had brain tumors in the left frontal lobe. Inclusion criteria for the patients were as follows: righthanded adults with a glioma located mainly in the SFG or the middle frontal gyrus who underwent awake surgery. The patients also underwent preoperative DTI. Left-sided dominance was confirmed by functional MRI or the Wada test. The study protocol was approved by the ethics committee of Nagoya University Graduate School of Medicine and Nagoya University Hospital. The patients had the appropriate cognitive function to understand and decide on the treatment and study protocol, and they provided written informed consent for their participation.

Neurological examination was performed before surgery. Handedness was assessed using the Edinburgh Handedness Inventory. Cognitive function including language was evaluated using the Standard Language Test of Aphasia (Japan Society for Higher Brain Dysfunction), Wechsler Adult Intelligence Scale-Third Edition, Wechsler Memory Scale-Revised, Frontal Assessment Battery, and Clinical Assessment for Attention. The mean age was 46.2 \pm 5.2 years (mean \pm SD). Patients' symptoms included seizures in 4 cases and headache, which was unrelated to the tumor, in 1 case. A preoperative examination of cognitive function revealed mild disturbances in all 5 cases. A summary of the patients' characteristics is shown in Table 1.

\section{DTI/Tractography, Preoperative Planning, and Intraoperative Navigation With Intraoperative MRI}

DTI was performed preoperatively in all cases using 3-T MRI ( $\mathrm{B}=2000,12$ directions; Siemens). The DTI data and anatomical MR images were sent to a planning workstation (iPLAN 2.6 and 3.0, Brainlab). The FAT was analyzed as follows: 1) regions of interest were set in the left SFG and in the pars triangularis and opercularis of the IFG, and 2) the tract between these 2 regions of interest was calculated and extracted with parameters set to 0.15 0.25 as the fractional anisotropy value and $15-20 \mathrm{~mm}$ as the minimum length value. The result was visualized as an "object," which was a representation of the extracted tract as the outline of the tract filled with an arbitrary color, on both the workstation and navigation. The superior longitudinal fascicles (SLFs), inferior frontooccipital fascicles (IFOFs), and pyramidal tract were also calculated and extracted with representations of each "object." All of the aforementioned tracts were identified and then overlaid onto the anatomical MR images. The tumor boundary was also identified and traced.

Surgery was performed under the guidance of navigation (Vector Vision Compact, Brainlab). Surgical planning data were sent to the navigator followed by the registration procedure. During this procedure, landmarks (usually 5-7) were acquired and were used to check the accuracy of the navigation system, and reregistration was performed with the landmarks when the accuracy was declined. Intraoperative MRI (APERTO Inspire, Hitachi) was performed when tumor removal was complete or the surgical goal was achieved according to the preoperative plans.

\section{Intraoperative Brain Mapping}

All patients underwent awake surgery with direct cortical stimulation. First, general anesthesia was administered with a laryngeal mask (i-gel, Intersurgical), followed by a wide craniotomy, which exposed the sylvian fissure, the frontal operculum including the precentral gyrus posteriorly, the SFG medially, and the middle temporal gyrus inferiorly. The tumor margins were verified by comparing the sulcal and gyral brain surface anatomy with a reconstructed 3D virtual image under the guidance of navigation. Somatosensory evoked potentials were also evaluated using a strip electrode (6 contacts) for electrophysiological determination of the central sulcus, where phase reversal of the N20 component is observed. Electrocorticography (ECoG) was performed during brain mapping and tumor removal by using 3 strip electrodes (18 contacts).

Cortical language mapping was performed during the awake portion of the surgery. A bipolar electrode (Unique Medical) with 5-mm tip spacing was used to apply electrical stimulation, with a biphasic current intensity between 2 and $8 \mathrm{~mA}(60-\mathrm{Hz}$ pulse frequency, 0.5 -msec single pulse phase, 8-second tissue contact; Neuromaster MEE1200, Nihon Kohden), while patients performed the tasks. The intraoperative language tasks consisted of naming pictures shown on a monitor connected to a personal computer (Panasonic). The patients were also asked to move their right arm and hand simultaneously, flex the right elbow while performing grasping movements of the right hand, and extend the elbow while opening the hand, all while naming the pictures. A speech therapist observed and evaluated the patients for symptoms such as speech arrest, dysarthria, anarthria, slowness of speech, facial movements, movement disturbances of the right arm, epilepsy, or any other symptoms related to patient safety. If the same response was obtained during at least 3 stimulations, a number tag was placed on that site. The optimal 
TABLE 1. Characteristics and findings of the 5 cases

\begin{tabular}{|c|c|c|c|c|c|c|c|}
\hline Case No. & $\begin{array}{l}\text { Age (yrs), } \\
\text { Sex }\end{array}$ & $\begin{array}{l}\text { Tumor } \\
\text { Location }\end{array}$ & $\begin{array}{l}\text { Path } \\
\text { Dx }\end{array}$ & $\begin{array}{l}\text { Primaryl } \\
\text { Rec }\end{array}$ & $\begin{array}{l}\text { Preop Language } \\
\text { Symptoms }\end{array}$ & $\begin{array}{l}\text { Tractography } \\
\text { of FAT }\end{array}$ & Subcortical Electrical Stimulation Results \\
\hline Case 1 & $45, \mathrm{M}$ & Lt SFG & DA & Primary & None & Yes & $\begin{array}{l}(+) \text { speech arrest; }(+) \text { repetition disturbance; } \\
(-) \text { orofacial apraxia }\end{array}$ \\
\hline Case 2 & $40, \mathrm{~F}$ & Lt SFG & Oligo & Primary & $\begin{array}{l}\text { Transient speech } \\
\text { disturbance }\end{array}$ & Yes & $\begin{array}{l}(+) \text { delayed speech initiation; }(+) \text { perseveration; } \\
(+) \text { repetition of the initial sound of a word }\end{array}$ \\
\hline Case 3 & $44, \mathrm{~F}$ & Lt MFG & Oligo & $\operatorname{Rec}$ & None & Yes & $(+)$ speech arrest \\
\hline Case 4 & $48, M$ & Lt SFG-IFG & GBM & $\operatorname{Rec}$ & None & Yes & $(+)$ speech arrest \\
\hline Case 5 & $54, \mathrm{M}$ & Lt SFG-IFG & $\mathrm{AO}$ & $\operatorname{Rec}$ & None & Yes & $(+)$ speech arrest; (+) naming disturbance \\
\hline
\end{tabular}

$\mathrm{AO}=$ anaplastic oligodendroglioma; DA = diffuse astrocytoma; GBM = glioblastoma; $\mathrm{MFG}$ = middle frontal gyrus; oligo = oligodendroglioma; path Dx = pathological diagnosis, rec = recurrence; $+=$ present; $-=$ absent.

threshold of current intensity was determined as the intensity that reproducibly elicited complete speech arrest when stimulating the ventral premotor cortex.

After determining the eloquent cortical areas, tumor removal was initiated. Tumor removal was performed using the gyrectomy approach. After the gyri that had been invaded by the tumor were removed, subcortical mapping was performed to determine the functional boundary in the deeper area. For subcortical mapping, the electrical stimulation parameters were the same as those for cortical mapping. We used the same intensity of stimulation as that used in Broca's area. Patients performed a picture-naming task or other tasks while flexing and extending their right arm.

\section{Determining the Locations of White Matter Symptoms}

The location of the stimulation was determined on the intraoperative MR image shown on the display of the navigation system. The intraoperative MR image was fused to the preoperative set of images on the iPLAN workstation, and these were used to evaluate the extent of brain shift, with adjustments of the location of interest for better accuracy. Two authors (M.F. and S.M.) agreed on the location. The adjustment procedure is described below.

The adjustment procedure was based on the stimulation point obtained on the intraoperative image, which generally exhibited certain distortion compared with the preoperative image. First, preoperative (T1-weighted and T2-weighted) and intraoperative (T1-weighted and T2-weighted) MR images were fused using the rigid registration algorithm that is provided in the iPLAN ("automatic image-fusion"). Using this procedure, the coordinates of each window became identical. Four image windows were then shown on the display, demonstrating the intraoperative images in the left column and the preoperative images in the right. Then the stimulation point was indicated as a crossbar on the intraoperative image (Fig. 1A). The shape and location of sulci, gyri, corticomedullary junctions, and tumor boundary/tumor resection boundaries around the crossbar were identified on both pre- and intraoperative images, and the images were compared. We also used operative findings, that is, which sulci were approached and which gyri were resected. If there was a shift of relevant anatomical structures, the distance from a certain common rigid point or a line (either an anatomical structure [e.g., falx cerebri] or an artificial line drawn on both pre- and intraoperative images in the same coordinate) was measured. The shift of the anatomical structures was assessed in 3 directions, namely, anteroposterior, mediolateral, and rostrocaudal. Based on the distance and direction of the shift, the crossbar on the preoperative image was moved to adjust the stimulation point. A representative case (Case 2) is shown in Fig. 1.

The surgical procedure during the awake state was also recorded using a multichannel simultaneous recording system, which recorded the operative field, the patient's face and voice, the ECoG, and the task image presented to the patient. The recorded multichannel image was also used to confirm the symptoms and locations offline. The influence of seizure activity on the symptoms by electrical stimulation was determined by checking the after-discharges on $\mathrm{ECoG}$, as well as by visual assessment of the patient.

\section{Results}

\section{DTI/Tractography}

Tractography of the FAT was successfully visualized in all 5 cases. The pathway originated from the SFG, posterior to the tumor, ran deep into the frontal lobe close to the lateral ventricle, and ended in the IFG. A depiction of FAT tractography in each case is shown in Fig. 2. In Case 2 , the tumor extended into the anterior and middle parts of the SFG and nearly reached the medial precentral sulcus. The FAT, however, was clearly demonstrated as extending from the small gyrus just anterior to the precentral gyrus to the IFG.

\section{Intraoperative Cortical and Subcortical Electrical Stimulation}

Table 1 summarizes the results of electrical stimulation. In all cases, positive findings were obtained during cortical stimulation of the M1 and the IFG. Cortical stimulation of the tumor area did not evoke any speech symptoms even when applying stimulation that was $2 \mathrm{~mA}$ greater than the amplitude used in cortical mapping of the M1 and the IFG (6-8 mA). In all cases, speech symptoms were observed during electrical stimulation in the area of the frontal white matter, mostly at the posterior part of the tumor, deep in the frontal white matter lateral to the superior frontal sulcus. In Cases 1, 3, 4, and 5, speech arrest occurred during stimulation. In Case 2, a disturbance of speech initiation was observed. Repetition was possible, but the volume of the voice was low. There were no motor disturbances of the extremities or tongue. The location of 
A

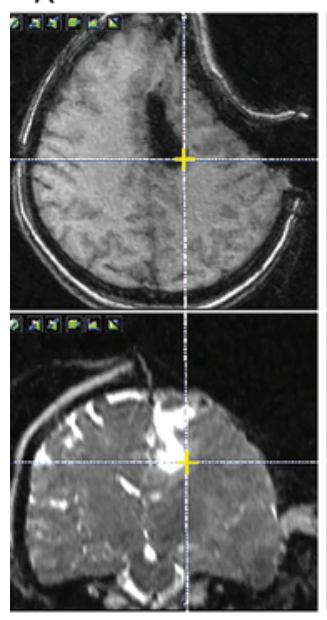

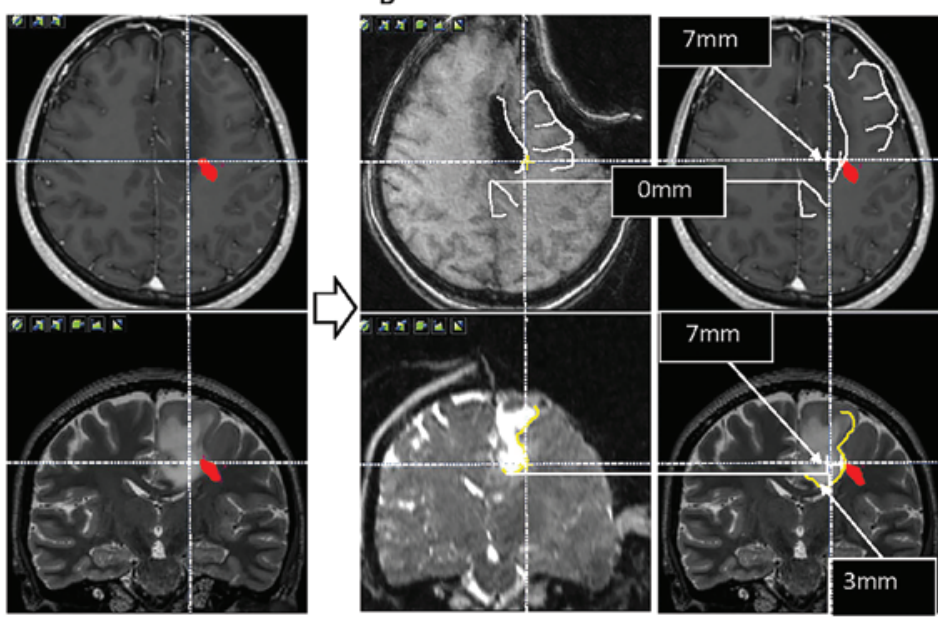

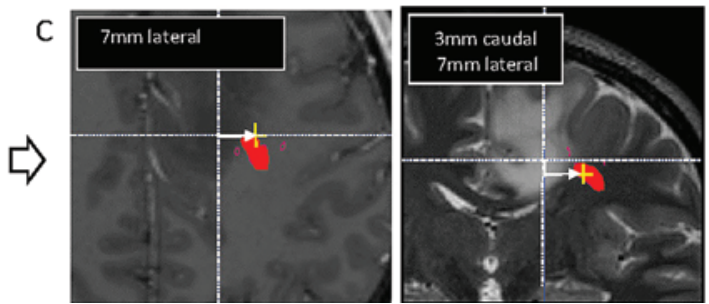

FIG. 1. Case 2. Representative case of the stimulation point adjustment from the intraoperative image onto the preoperative image. A: Four windows of iPLAN cranial software are indicated. Intraoperative axial T1-weighted image (upper left), intraoperative coronal T2-weighted image (lower left), preoperative axial T1-weighted image (upper right), and preoperative coronal T2-weight image (lower right). All images were fused by the rigid registration algorithm provided by IPLAN and have the same coordinates. The yellow crossbars show the stimulation point. The objects of the FAT, created with preoperative DTI, were overlaid onto preoperative images (upper and lower right). Note that the crossbar on the preoperative images (upper and lower right) seemed to be located inside the tumor, although the tumor had already been resected. B: Extent of the brain shift near the stimulation point was evaluated using anatomical landmarks and measured in $\mathrm{x}-\mathrm{y}-\mathrm{z}$ coordinates ( $\mathrm{x}$, mediolateral; $\mathrm{y}$, anteroposterior; $\mathrm{z}$, rostrocaudal). The stimulation point was located at the deep posterolateral aspect of the resection cavity (delineated by the white curved line). Note that the surface and sulci of the frontal lobe (white line) were shifted medially, suggesting that there was an apparent brain shift on the intraoperative image toward the medial direction. The distance between the crossbar and the boundary of the tumor was measured as $7 \mathrm{~mm}$ ( $x$ axis). A sulcus located in the posterior area of the resection cavity, running slantwise from the medal aspect of the brain in a posterolateral direction, had no apparent shift $(0 \mathrm{~mm}$, y axis). The amount of the shift in the $z$ axis was evaluated on the coronal images. Comparison between the bottom of the resection cavity on the intraoperative image and the tumor boundary on the preoperative image (both of them delineated by a yellow line) revealed a 3-mm upward shift in the z-axis direction. C: According to the evaluation above, the amount of the brain shift near the stimulation point was considered as $7 \mathrm{~mm}$ medial ( $x$ axis) and $3 \mathrm{~mm}$ rostral ( $z$ axis). The shift in the y axis was $0 \mathrm{~mm}$. Therefore, we concluded that due to the brain shift, the stimulation point on the intraoperative image should be adjusted and moved $7 \mathrm{~mm}$ lateral and $3 \mathrm{~mm}$ caudal on the preoperative image. Note that the stimulation point is very close to the FAT (red areas).

the stimulation point of all cases, as checked by the navigation system and intraoperative MRI, was close (within $3 \mathrm{~mm}$ ) to the tractography of the FAT (Fig. 2). Figure 3 shows the 3 locations of electrical stimulation on intraoperative MR images with remarkable brain shift, as well as the corrected locations on preoperative MR images with the "object" of the FAT. In Case 5, speech arrest was observed at 3 points, which were located longitudinally along the tract on its anterior surface (Figs. 3 and 4). In all patients, gross-total resection of the tumor was achieved without any severe language problems on the Standard Language Test of Aphasia examination, which was performed 10-14 days after surgery.

\section{Discussion}

\section{Deep Frontal White Matter Tracts and Language Functions}

Recent studies have shown that the language system is not as simple as the classic model, which consists of Broca's area located in the IFG, Wernicke's area located in and around the STG, and the arcuate fascicle, which connects the 2 areas. ${ }^{6}$ Recently, a better understanding of the neural basis of language has been established via models that include many cortical areas working together as part of a larger network, such as the SFG, inferior parietal lobule, middle temporal gyrus, bottom area of the temporal lobe, and other white matter tracts such as the SLFs, IFOFs, and fibers in the deep frontal lobe. ${ }^{6,10,11}$ The SMA is known to play an important role in speech initiation and spontaneous speech. ${ }^{1}$ Intrafrontal networks, especially fibers connecting the SMA and Broca's area, are also considered to support the aforementioned functions. ${ }^{17}$ Lesions within the network, either the SMA or the deep frontal white matter, cause transcortical motor aphasia. ${ }^{2,8}$ In fact, we have observed postoperative speech deterioration, especially disturbances of speech initiation and speech spontaneity 

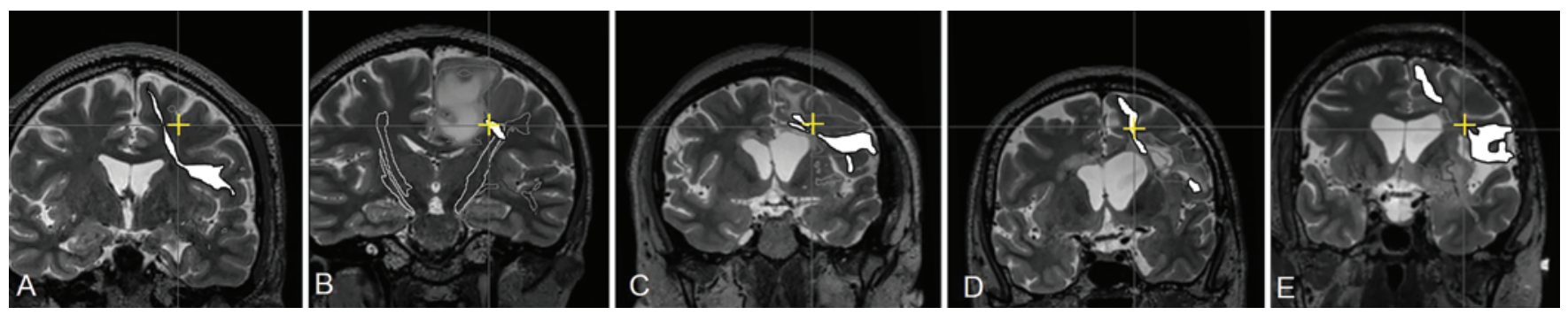

FIG. 2. Preoperative coronal MR images in Cases 1-5, with the 3D objects of the FAT, formed by DTI tractography (white areas outlined by black). The yellow crosses show sites that responded positively to electrical stimulation during subcortical mapping in awake surgery. Figure is available in color online only.

(transcortical motor aphasia), in cases in which the tumor was removed from the deep frontal lobe of the dominant side and the SFG and IFG were preserved in both cortices (data not shown). Such observations motivated us to study this particular tract. In this study, we observed reproducible language symptoms in all 5 patients during electrical stimulation of the frontal white matter. We also examined the patients' symptoms with electrical stimulation at the white matter sites. Our results showed that patients displayed language symptoms without any motor disturbances of the extremities or tongue, suggesting that the fibers are not simply related to motor functions.

Several reports have demonstrated the existence of this pathway using structural imaging, such as DTI tractography. ${ }^{7,9,15,18}$ However, there is controversy concerning the detailed anatomy of the white matter tract. Several tractography studies have demonstrated the existence of a deep frontal tract connecting the SMA and pre-SMA to Broca's area. . $7,7,12,15,18$ However, a fiber dissection study revealed that the fibers connect Broca's area to the lateral SFG, not to the SMA. ${ }^{18}$ Catani et al. ${ }^{3}$ studied the tract using both tractography and fiber dissection of postmortem brains. The tractography results showed that the tract ran from the IFG (pars opercularlis and pars triangularis) to both the lateral and medial parts of the SFG. ${ }^{3}$ On the other hand, fiber dissection failed to show the tract. ${ }^{3}$ It is too early to draw a conclusion about whether the tract terminates in the medial or lateral side of the SFG, or both. Here, we used the term "frontal aslant tract" to refer to the fiber tracts connecting the SFG, including both the lateral and medial parts, and the IFG (Broca's area, pars opercularis and triangularis). Our focus was on determining the functional aspects of the frontal white matter.

\section{Identification of the FAT}

Determining the exact location of the stimulation
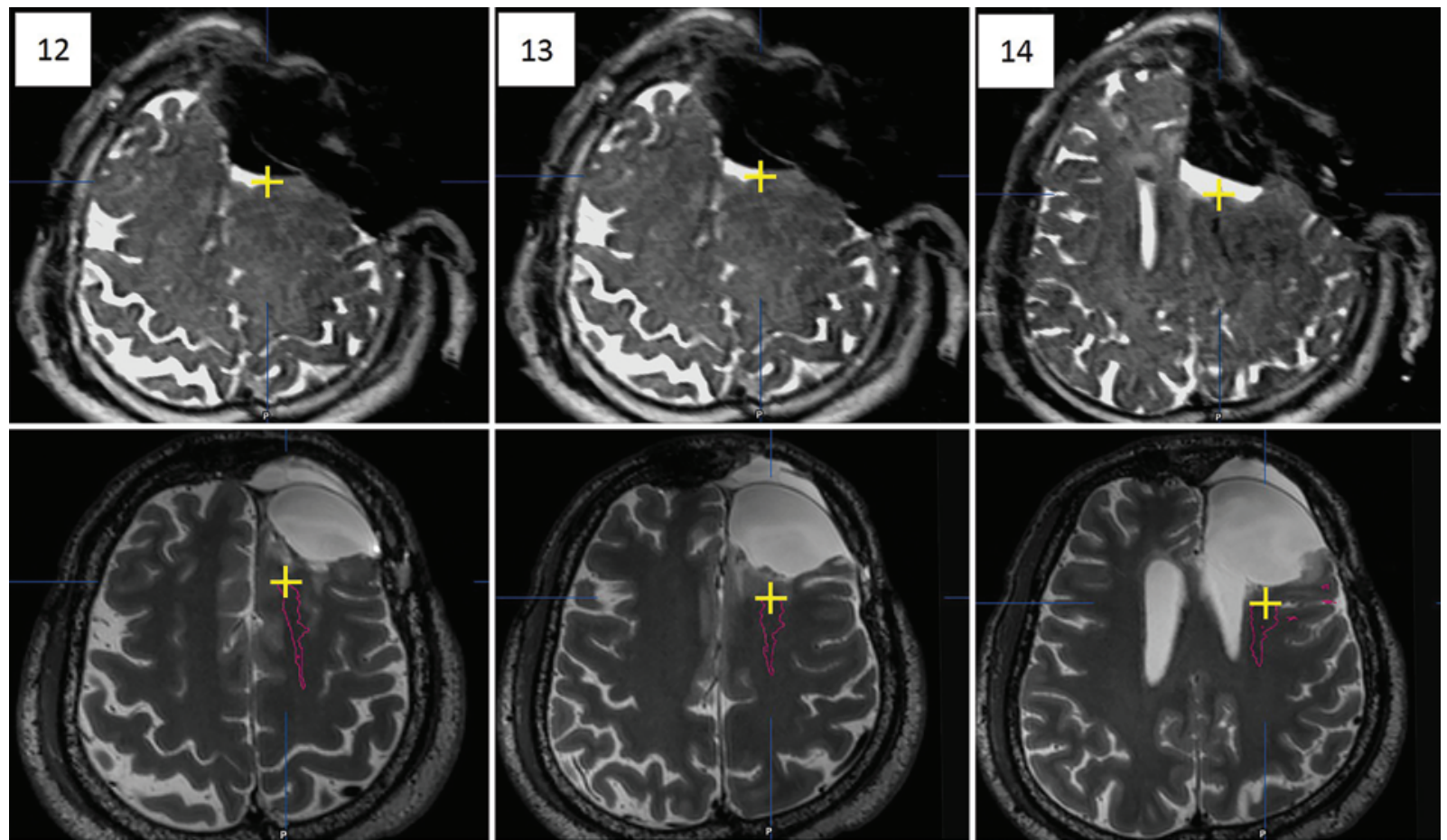

FIG. 3. Subcortical stimulation sites at Tags 12, 13, and 14 from Fig. 4 are indicated on intraoperative T2-weighted images (upper row, yellow crosses). The corresponding points were estimated by correcting the intraoperative brain shift using fused intraoperative and preoperative MR images. The estimated stimulation sites on the preoperative images with the frontal aslant tract are shown in the lower row (yellow crosses). Figure is available in color online only. 

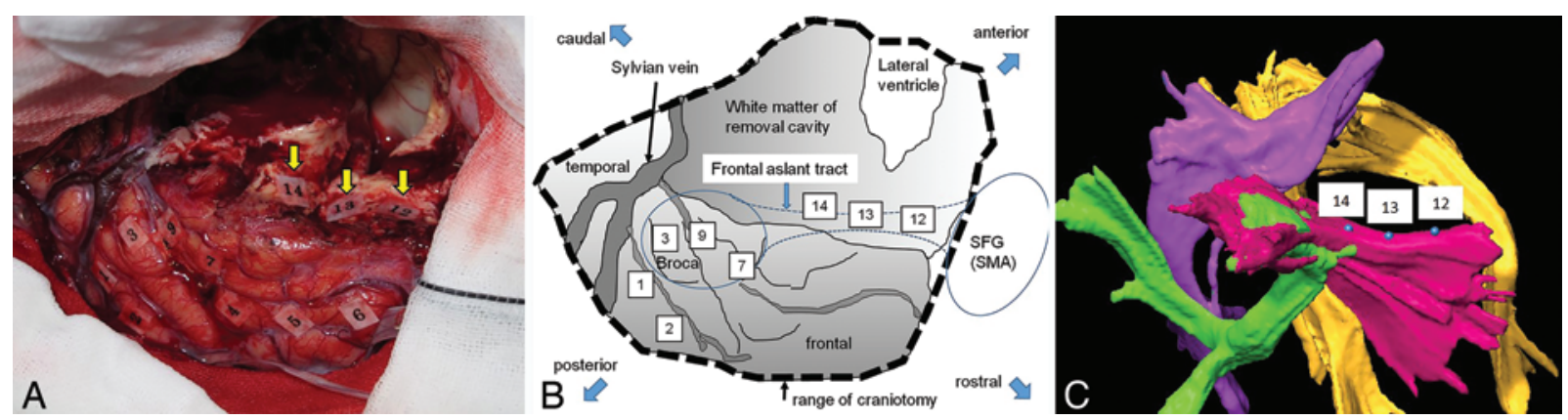

FIG. 4. Case 5. Intraoperative photograph (A), diagram (B), and 3D view of the tractography (C). Cortical mapping showed the primary motor cortex (Tags 1 and 2) and Broca's area (Tags 3, 9, and 7). Subcortical mapping demonstrated positive language symptoms during electrical stimulation at Tags 12, 13, and 14 (yellow arrows). All of these sites were considered adjacent to the frontal aslant tract, based on preoperative DTI examinations and navigation corrected by intraoperative MRI. Anatomical relationships are shown in the diagram (B). The subcortical stimulation sites were overlaid onto a 3D view of the tractography $(C)$, demonstrating that the 3 sites are aligned on the anterior aspect of the object of the frontal aslant tract (pink). green = SLF; purple = IFOF; yellow = corticospinal tract (bilateral).

point is an important issue. It is especially difficult to identify the exact location of white matter areas because no clear landmarks exist, such as vessels or the shape of the cortex. In addition, brain shifts often occur because of cerebrospinal fluid drainage, mass reduction of the tumor, and retraction due to surgical manipulation. ${ }^{16}$ We conducted image-guided awake surgery using a navigation system and intraoperative MRI. During surgery, the navigation system was able to demonstrate the "object" of the FAT, created with preoperative DTI using the navigation workstation.

We were also able to demonstrate the corticospinal tract, SLFs, and IFOFs, which allowed us to determine the location of the stimulation point and the spatial relationship between the point and the above white matter tracts. The influence of the brain shift was evaluated using intraoperative MR images. Therefore, the methodological design of our study has an advantage over other studies in which conventional methods are used because we were more precisely able to identify the exact location of electrical stimulation. To our knowledge, we are the first to use a navigation system with the support of intraoperative MRI to determine the precise location of stimulation, and we strongly believe that the stimulation sites were on or adjacent to the FAT. It could be argued, however, that the symptoms elicited by stimulation were due to the co-stimulation of other tracts. Long association or projection tracts, such as the SLFs, IFOFs, pyramidal tract, and subcallosal fasciculus, could be candidates for producing the observed speech symptoms in the dominant frontal lobe. ${ }^{6}$ All of these fiber tracts, however, run apart from the FAT, at least in its mid- and superomedial portion. Thus, the symptoms induced by stimulation cannot be explained only by co-stimulated fiber tracts in place of the FAT, which is located very close to the stimulation point. In Case 5, positive findings were noted at 3 stimulation points: Tags 12, 13, and 14 along with the FAT (Fig. 3). Of the 3 points, Tags 12 and 13 were located far from the SLF and other candidate fibers. Tag 14, however, could be close to the SLF, which enters the IFG together with the FAT. In Case 2, the pyramidal tract ran relatively close to the stimulation points. This patient, however, did not show any orofacial movement deficits, and she did not exhibit im- pairments in her right upper or lower extremity; however, speech dysfunction was obvious. Therefore, the resulting symptoms caused by stimulation were not induced by activation of the pyramidal tract. The subcallosal fasciculus, which connects frontomesial structures to the striatum, ran medial to the stimulation points in Cases 1,2,3, and 5 . Commissural fibers of the corpus callosum are extensive in their connection of the left and right hemispheres and may be co-stimulated with the FAT; thus, this possibility cannot be excluded in the current study.

\section{Consideration of Interindividual Variability or Plasticity of the Neural Basis of Language}

It has been reported that there is significant interindividual variability of cortical language localization, which could be due to reorganization induced by slow-growing tumors or other mechanisms. Cortical reorganization might also alter the function of associating white matter tracts. If a patient's cortical language localization significantly differs from the typical localization, the result of white matter stimulation might vary. Thus, the seed area for tractography should be placed accordingly, taking into consideration the localization of both the classical and the aberrant tract. In all 5 patients in this study, however, the anterior language area (Broca's area) was determined by electrophysiological mapping at the posterior inferior frontal gyri (pars triangularis and/or pars opercularis), and speech arrest occurred when stimulating the ventral precentral gyrus in all cases. Moreover, DTI tractography successfully depicted the FAT in all cases, although the fiber did run differently in the frontal lobe of each patient due to tumor existence. We assume that the FAT was still intact without reorganization in all cases included in this study, since the FAT is the association fiber connecting the posterior IFG to the ventral precentral gyrus. This could be why the results were very much reproducible.

\section{Resectability of Tumors in the FAT}

In our cases, the resection boundary was determined by the appearance of language symptoms during electrical stimulation. Fortunately, the region of the FAT identified 
using electrical stimulation did not show tumor invasion on MR images; therefore, we were able to preserve these areas. Postoperatively, none of the patients exhibited apparent deterioration in speech function. Controversy remains regarding whether the FAT should be preserved, namely, whether the aphasia caused by destruction of the dominant FAT is a permanent or transient symptom. When a positive response is observed during electrical stimulation of the tract and tumor invasion is apparent, one may consider removing it. In such cases, postoperative symptoms will appear. However, these symptoms might be transient and improve over time, similar to the symptoms after removal of the SMA. The transcortical motor aphasia known to appear after resection of SMA tumors recovers within several weeks in most cases..$^{14,19}$ Krainik et al.$^{13}$ reported that the functional recovery occurred with activation of the contralateral SMA. If this is true, commissural fibers from the contralateral SMA might play a crucial functional role and thus should be preserved. This is an important issue, which should be continuously discussed, particularly in regard to the balance between oncological control of the tumor and functional preservation of the patients.

\section{Conclusions}

We identified the FAT preoperatively by DTI tractography and confirmed the tract intraoperatively using electrical stimulation during awake surgery. Similar to the SMA, the FAT is assumed to play an important role in speech initiation and spontaneity by connecting Broca's area and the SFG. It is not conclusive whether this tract should be preferentially preserved. Further evaluation is necessary to resolve this issue, particularly considering the balance between oncological tumor control and functional preservation. However, the pathway should be recognized in the clinical setting of brain tumor surgery, especially for tumors located in the deep frontal lobe of the languagedominant side.

\section{Acknowledgments}

We thank Daisuke Hara, Maki Tobinaga, and Junko Sugiura (Department of Rehabilitation, Nagoya University Hospital) for evaluations of language and cognitive function of the patients.

\section{References}

1. Alario FX, Chainay H, Lehericy S, Cohen L: The role of the supplementary motor area (SMA) in word production. Brain Res 1076:129-143, 2006

2. Alexander MP, Benson DF, Stuss DT: Frontal lobes and language. Brain Lang 37:656-691, 1989

3. Catani M, Dell'acqua F, Vergani F, Malik F, Hodge H, Roy P, et al: Short frontal lobe connections of the human brain. Cortex 48:273-291, 2012

4. Catani M, Mesulam MM, Jakobsen E, Malik F, Martersteck A, Wieneke C, et al: A novel frontal pathway underlies verbal fluency in primary progressive aphasia. Brain 136:26192628, 2013

5. Duffau H, Gatignol P, Mandonnet E, Capelle L, Taillandier L: Intraoperative subcortical stimulation mapping of language pathways in a consecutive series of 115 patients with Grade II glioma in the left dominant hemisphere. J Neurosurg 109:461-471, 2008
6. Fernández Coello A, Moritz-Gasser S, Martino J, Martinoni M, Matsuda R, Duffau H: Selection of intraoperative tasks for awake mapping based on relationships between tumor location and functional networks. J Neurosurg 119:13801394,2013

7. Ford A, McGregor KM, Case K, Crosson B, White KD: Structural connectivity of Broca's area and medial frontal cortex. Neuroimage 52:1230-1237, 2010

8. Freedman M, Alexander MP, Naeser MA: Anatomic basis of transcortical motor aphasia. Neurology 34:409-417, 1984

9. Guevara P, Poupon C, Rivière D, Cointepas Y, Descoteaux $\mathrm{M}$, Thirion B, et al: Robust clustering of massive tractography datasets. Neuroimage 54:1975-1993, 2011

10. Hickok G: The cortical organization of speech processing: feedback control and predictive coding the context of a dualstream model. J Commun Disord 45:393-402, 2012

11. Hickok G, Poeppel D: Dorsal and ventral streams: a framework for understanding aspects of the functional anatomy of language. Cognition 92:67-99, 2004

12. Kinoshita M, Shinohara H, Hori O, Ozaki N, Ueda F, Nakada $\mathrm{M}$, et al: Association fibers connecting the Broca center and the lateral superior frontal gyrus: a microsurgical and tractographic anatomy. J Neurosurg 116:323-330, 2012

13. Krainik A, Duffau H, Capelle L, Cornu P, Boch AL, Mangin JF, et al: Role of the healthy hemisphere in recovery after resection of the supplementary motor area. Neurology 62:1323-1332, 2004

14. Laplane D, Talairach J, Meininger V, Bancaud J, Orgogozo JM: Clinical consequences of corticectomies involving the supplementary motor area in man. J Neurol Sci 34:301-314, 1977

15. Lawes IN, Barrick TR, Murugam V, Spierings N, Evans DR, Song M, et al: Atlas-based segmentation of white matter tracts of the human brain using diffusion tensor tractography and comparison with classical dissection. Neuroimage 39:62-79, 2008

16. Maesawa S, Fujii M, Nakahara N, Watanabe T, Wakabayashi $\mathrm{T}$, Yoshida J: Intraoperative tractography and motor evoked potential (MEP) monitoring in surgery for gliomas around the corticospinal tract. World Neurosurg 74:153-161, 2010

17. Morgan VL, Mishra A, Newton AT, Gore JC, Ding Z: Integrating functional and diffusion magnetic resonance imaging for analysis of structure-function relationship in the human language network. PLoS One 4:e6660, 2009

18. Oishi K, Zilles K, Amunts K, Faria A, Jiang H, Li X, et al: Human brain white matter atlas: identification and assignment of common anatomical structures in superficial white matter. Neuroimage 43:447-457, 2008

19. Rostomily RC, Berger MS, Ojemann GA, Lettich E: Postoperative deficits and functional recovery following removal of tumors involving the dominant hemisphere supplementary motor area. J Neurosurg 75:62-68, 1991

\section{Author Contributions}

Conception and design: Fujii, Maesawa, Futamura. Acquisition of data: Fujii, Maesawa, Motomura, Futamura, Hayashi, Koba. Analysis and interpretation of data: Fujii, Maesawa, Futamura, Hayashi, Koba. Drafting the article: Fujii. Critically revising the article: Maesawa. Approved the final version of the manuscript on behalf of all authors: Fujii. Administrative/technical/material support: Maesawa, Futamura, Wakabayashi. Study supervision: Fujii, Wakabayashi.

\section{Correspondence}

Masazumi Fujii, 65 Tsurumai-cho, Showa-ku, Nagoya 466-8550, Japan.email: fujiim@med.nagoya-u.ac.jp. 